\title{
Possible Nuclear Structure Effects in Even-Even Osmium Nuclei
}

\author{
E. Bashandy and M. S. El-Nesr \\ Nuclear Physics Department, Atomic Energy Establishment, Cairo, Egypt
}

(Z. Naturforsch. 29 a, 1125-1130 [1974] ; received March 26, 1974)

\begin{abstract}
A compilation of the multipole mixing of $2^{\prime}+\rightarrow 2+$ transitions in ${ }^{186} \mathrm{Os},{ }^{188} \mathrm{Os},{ }^{190} \mathrm{Os}$ and ${ }^{192} \mathrm{Os}$ is given. In view of recent results obtained at our laboratory, indicating large anomalies in the conversion process of inhibited Ml transitions, the study has been extended for even-even Osmium nuclei. A series of experiments has been performed in which the conversion coefficients and transition probabilities were measured. The data of $2^{\prime}+\rightarrow 2+$ transitions were analysed by taking into account nuclear structure effects on the Ml internal conversion process. The Ml admixtures obtained are compared with Greiner's calculations. Values of reduced transition probabilities B $(\mathrm{E} 2,0 \rightarrow 2+)$, B (E2, $\left.2^{\prime}+\rightarrow 2+\right)$, the mixing ratio $\delta=\left\langle 2\|\mathrm{E} 2\| 2^{\prime}\right\rangle \mid\left\langle 2\|\mathrm{M} 1\| 2^{\prime}\right\rangle$ and the transition branching ratio $\mathrm{T}\left(2^{\prime} \rightarrow 2\right) / \mathrm{T}\left(2^{\prime} \rightarrow 0\right)$ are reported for second and higher $2+$ states. The results are compared to the pairing-plus-quadrupole model calculations of Kumar and Baranger.
\end{abstract}

\section{Introduction}

Recent development of microscopic description (based on the many-body theory) has made studies of some none-phonon features of excited states in doubly even nuclei of special importance. The nuclei of Osmium and Platinum form part of a transition region in which the shape of the nuclear surface turns from spherical to deformed. Nuclei in this region are therefore particularly suited for a critical test of nuclear models. From this point of view, Platinum and Osmium nuclei are very interesting isotopes ${ }^{1}$. Though there appears to be a systematic trend of level energies, no simple trend is found in probabilities of the Ml transitions of $2^{\prime}+\rightarrow 2+$. Systematic searches for the E0 component in the $2^{\prime}+\rightarrow 2+$ transition and for the penetration or the dynamic effect in the Ml internal conversion would supply very crucial information ${ }^{2,3}$. In order to evaluate such possibilities, an accurate knowledge is required for the E2: M1 mixing ratio. Detailed studies on ${ }^{192} \mathrm{Pt},{ }^{194} \mathrm{Pt}$ and ${ }^{196} \mathrm{Pt}$ have been reported by the author ${ }^{4,5,6}$ and valuable conclusions could be drawn.

The present study was started to confirm our previous results ${ }^{5}$ on even-even $\mathrm{Pt}$ isotopes. Eveneven Osmium isotopes were under several experimental investigations by the author ${ }^{7,8,9}$, however, no attention has been given to determine the nuclear structure effects. The studies presented in this work were concentrated on the conversion coefficients and the mixing ratios of the $2^{\prime}+\rightarrow 2+$ transitions in ${ }^{186} \mathrm{Os},{ }^{188} \mathrm{Os},{ }^{190} \mathrm{Os}$ and ${ }^{192} \mathrm{Os}$. Kumar and Baranger ${ }^{10}$, and Kumar ${ }^{11}$ have calculated an extensive set of nuclear properties for the doubly even nuclei of $\mathrm{W}, \mathrm{Os}$ and $\mathrm{Pt}$ from the pairing-plus-quadrupole model. These properties include electric-quadrupole and magnetic-dipole transition matrix elements as well as static quadrupole moments of excited states. Since these model calculations are the most advanced and explicitly incorporate collective modes, a comparison to these predictions is of special interest.

The presence of an electric monopole component in the transition between the second $2^{\prime}+$ state and the first $2+$ state $\left(2^{\prime}+\rightarrow 2+\right)$ in even nuclei was for the first time pointed out by Church and Weneser ${ }^{12}$, who estimated the strength of this component for the case of ${ }^{196} \mathrm{Pt}$ and ${ }^{198} \mathrm{Hg}$ on the basis of the experimental conversion coefficient and mixing ratio M1/E2 available at that time and compared it with the values expected from the "free-vibration" model ${ }^{13}$ and the "shape-unstable" model ${ }^{14}$. For an accurate measurement of this component, an electron-gamma angular correlation experiment has been proposed ${ }^{4}$, because of appearance of the linear term of an E0matrix element in the coefficient $A_{2}$ of the angular correlation function. In analysing experimental data, one should be very careful in taking into account the dynamic effect of finite nuclear size on the Ml conversion process ${ }^{12}$. It can be expressed as the ratio of the penetration matrix element for the K-conversion of the Ml transition to the normal Ml gamma-ray matrix element, that is $m_{\mathrm{e}} / m_{\gamma}$. Since the monopole matrix element is fairly sensitive to the finer details of nuclear wave functions, measurement of its value may provide an important clue for understanding the excitation properties of the first and second $2+$ excited states in even nuclei. The physical content of 
this problem is similar to that which arises in the case of the Ml component in the previously studied transitions ${ }^{4,5}$. A series of experiments have been performed in which the K-conversion coefficient and transition probabilities were measured by the author and others ${ }^{4,9}$. The intention was to find the relative contribution of the penetration matrix elements for transitions forbidden by different selection rules. Hence, the strongly retarded Ml transitions in eveneven Osmium isotopes were investigated. It was shown that the internal conversion matrix elements are influenced appreciably by penetration effects.

\section{Experimental Procedure}

In this contribution we present the status of the analysis of our results from the study of $2^{\prime}+\rightarrow 2+$ transitions characteristics in the series of even neutron deficient Osmium nuclei, Figure 1. A knowl-
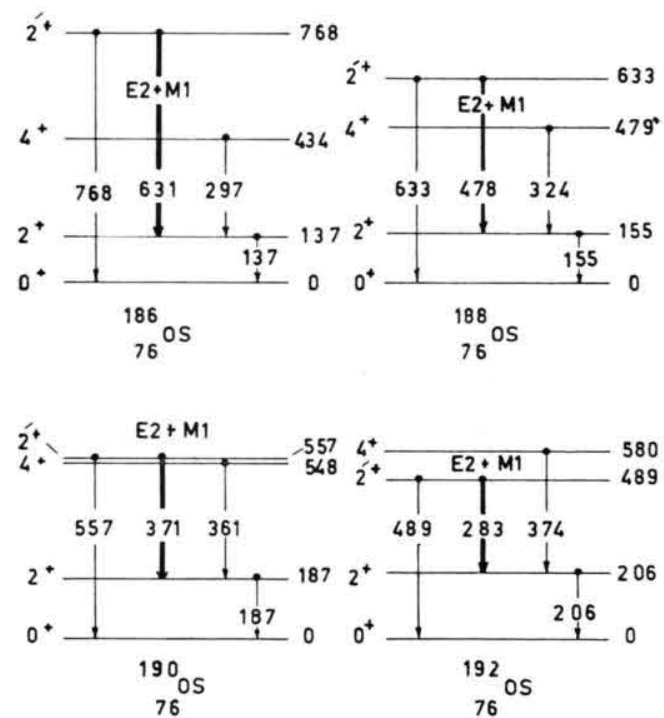

Fig. 1. Level diagrams of states of ${ }^{186} \mathrm{Os},{ }^{188} \mathrm{Os},{ }^{190} \mathrm{Os}$ and ${ }^{192}$ Os.

edge of conversion coefficients, mixing ratio E2/M1, and transition probability, is necessary. The nuclear spectroscopic equipment at our disposal consist of two types of beta-ray spectrometers, a double focusing and a double lens electron-electron magnetic spectrometer. A description of these instruments was published elsewhere ${ }^{7,8,9}$.

The measurements were performed applying different techniques, in order to determine the above mentioned parameters. The studies carried out on ${ }^{186} \mathrm{Os}$ and ${ }^{188} \mathrm{Os}$ were based on the decays of ${ }^{186} \mathrm{Re}$ and ${ }^{188} \mathrm{Re}$ respectively, while the measurements on ${ }^{192} \mathrm{Os}$ was based on the decay of ${ }^{192} \mathrm{Ir}$. Radioactive rhenium was produced by thermal neutron bombardment of rhenium oxide of natural isotopic abundance as well as of rhenium enriched to $99 \%{ }^{185} \mathrm{Re}$. Radioactive iridium was also produced by thermal neutron bombardment of enriched ${ }^{191} \mathrm{Ir}$. The flux was about $10^{13}$ neutrons $/ \mathrm{cm}^{2}$ sec. For the internal conversion studies the radioactive material was uniformly sputtered on aluminium foil of thickness $\sim 0.7 \mathrm{mg} /$ $\mathrm{cm}^{2}$. The sputtered material was distributed in a rectangular form of dimensions $0.2 \times 2 \mathrm{~cm}^{2}$. For the external conversion source, the active material was enclosed in a spectroscopically pure aluminium capsule. A uranium converter $3 \mathrm{mg} / \mathrm{cm}^{2}\left(5 \times 30 \mathrm{~mm}^{2}\right)$ was used. A point sources of $4 \mathrm{~mm}$ diameter was prepared for the electron-electron coincidence spectrometer.

The decay schemes of even Osmium nuclei have been established mainly on the basis of single gamma spectra, gamma-gamma coincidences and conversion electron data and by using energy sum relations ${ }^{9}$. The gamma transitions under investigation are the 631,478 and $283 \mathrm{keV}$ gamma rays in ${ }^{186} \mathrm{Os}$, ${ }^{188} \mathrm{Os}$ and ${ }^{192} \mathrm{Os}$ respectively. The coincidence measurements were recorded between Beta-conversion electrons of $631 \mathrm{keV}$ gamma, Beta-conversion electrons of $478 \mathrm{keV}$ gamma, conversion electrons of $201 \mathrm{keV}$ gamma-conversion electrons of $283 \mathrm{keV}$ gamma, conversion electrons of $631 \mathrm{keV}$ gammaconversion electrons of $137 \mathrm{keV}$ gamma, conversion electrons of $478 \mathrm{keV}$ gamma-conversion electrons of $155 \mathrm{keV}$ gamma and conversion electrons of $283 \mathrm{keV}$ gamma-conversion electrons of $206 \mathrm{keV}$ gamma, see Figure 1.

The internal-external conversion method for the experimental determination of internal conversion coefficients was used ${ }^{7}$. This method is based on straightforward measurements of the rates of emission of internal conversion electrons and gamma rays belonging to the same transition. The ratio of these rates defines the internal conversion coefficient. If we let $A_{\text {in }}$ and $A_{\mathrm{ex}}$ denote the intensities of conversion electrons and photoelectrons, respectively, it can easily be shown that the internal conversion coefficient $\alpha_{i}$ is given by:

$$
\alpha_{i}=\left[\left(A_{\mathrm{in}}\right)_{i} /\left(A_{\mathrm{ex}}\right)_{j}\right] \tau_{i} f_{j} k d b q .
$$

The internal conversion takes place in the $i^{\text {th }}$ shell or subshell and the external conversion in the $j^{\text {th }}$ shell or subshell. The quantity $k$ is the relative source strength if different radioactive sources are employed for internal conversion and external conversion, $d$ 
the thickness of the converter (usually expressed in $\left.\mathrm{mg} / \mathrm{cm}^{2}\right), b$ a dimensional factor and $q$ the relative instrumental transmission if the latter is not the same for internal conversion and external conversion. The basic quantity for the determination of the internal conversion coefficient is the integrated photoelectric cross section $\tau$. It is therefore essential to have access to accurate tables of $\tau$. At present the most accurate calculations are those for $\tau_{K}$ by Hultberg, Nagel and Olsson ${ }^{15}$. They are corrected to any order in $\alpha_{\mathrm{z}}$ but neglect the effect of screening. The correction for the latter effect is rather small for the K-shell and can partially be corrected for. A rectangular source and converter is the most useful geometry for measurements with a magnetic spectrometer of the double-focusing type. Correction factors $f_{\mathrm{K}}$ including all the above mentioned effects are computed by means of the electronic computer BESK at Stockholm for 13 gamma energies from 159 to $5000 \mathrm{keV}$ for the specific geometry used in the present experiment. In the present work the method was employed without numerical calculation of $k, d, b$, and $q$, by comparing the equation above for a transition in the same source with known $\alpha_{K}$ with the transition of unknown $\alpha_{K}$ to be measured. The comparison was made using the known Kconversion coefficient $\alpha^{n}$. The K-conversion coefficient $\alpha_{\mathrm{K}}(x)$ of any transition was thus determined as:

$$
\alpha_{\mathrm{K}}(x)=\alpha_{\mathrm{K}}{ }^{n} \frac{A_{\mathrm{in}}^{x} A_{\mathrm{ex}}^{n} \tau_{\mathrm{K}}(x) f(x)}{A_{\mathrm{in}}^{n} A_{\mathrm{ex}}^{x} \tau_{\mathrm{K}}^{n} f(n)} .
$$

This comparative method of determining the internal conversion coefficient is straightforward. It does not depend upon any knowledge of the decay scheme and it shows great advantages over other methods in cases where the decay is complicated. The K-conversion probabilities of some transitions were determined from the electron-electron coincidence measurements ${ }^{8}$. In the experiments the K-conversion electron of the transition in question was focused in one channel while the $\mathrm{K}$ or $\mathrm{L}$-conversion electrons of known transition were recorded in coincidence alternatively. The coincidence counting rate $N_{\mathrm{c}}$ can be written as,

$$
N_{\mathrm{c}}=N_{1} \delta_{1} H_{2} f_{2} W_{2} \xi_{2} \varepsilon_{\mathrm{c}} \gamma
$$

where, $H_{2} \quad=$ conversion probability of transition 2,

$f_{2}=$ fraction of the conversion line focused in spectrometer 2 ,

$$
\begin{aligned}
W_{2} \quad= & \text { transmission in spectrometer } 2, \\
& \text { and } \\
\xi_{2} \quad= & \text { efficiency of the detector in spec. } \\
& \text { trometer } 2 .
\end{aligned}
$$

In our calculations, $\xi_{2}$ has been set equal to unity while the efficiency of the coincidence circuit $\varepsilon_{\mathrm{c}}$ was found to be 90 percent, and assumed to be constant during the experiments. The coincidence correlation constant $\gamma$ was considered to be one, since the aperture angle in each spectrometer is large. The transmission of the two spectrometers has been systematically investigated for different source diameters, and baffle settings ${ }^{8}$. The conversion probability $H$ for the transition in question has been calculated as

$$
H_{\mathrm{K}}=\alpha_{\mathrm{K}} /\left(1+\alpha_{\mathrm{tot}}\right) \text {. }
$$

The K-conversion coefficient can be given by

where

$$
\alpha_{\mathrm{K}}=H_{\mathrm{K}} /\left(1-H_{\mathrm{tot}}\right)
$$

$$
H_{\mathrm{tot}}=H_{\mathrm{K}}(1+L / K+M / K)
$$

and the total conversion coefficient $\alpha_{\text {tot }}$ was taken to be

$$
\alpha_{\text {tot }}=\alpha_{K}+1.33\left(\alpha_{L_{1}}+\alpha_{L_{11}}+\alpha_{L_{11}}\right)
$$

In the interpretation of internal conversion data, highly converted transitions between even parity states with the same angular momentum have been assumed to be $\mathrm{E} 0$ or $\mathrm{E} 0+\mathrm{E} 2+\mathrm{M} 1$.

We have also measured ${ }^{7}$ some halflives of the $2^{+}$levels. A magnetic electron-electron coincidence spectrometer set at a resolution and transmission of $2.5 \%$ was used with plastic scintillators. In the experiments we recorded coincidences between the beta branch from the ground state of Re feeding levels in $\mathrm{Os}$ and the $\mathrm{K}$ or L-conversion electrons of the transition in question.

Values of $\mathrm{B}\left(\mathrm{E} 2,2^{\prime}+\rightarrow 0\right)$ have been deduced and compared with those calculated by Kumar and Baranger ${ }^{10}$, see Table 1 . The mixing ratio $\delta$ has been calculated from the conversion coefficient data as,

$$
\delta=\sqrt{\frac{a^{\mathrm{K}}(\mathrm{M} 1)-\alpha^{\mathrm{K}}(\exp )}{\alpha^{\mathrm{K}}(\exp )-\alpha^{\mathrm{K}}(\mathrm{E} 2)}}
$$

which is also defined as,

$$
\delta=\sqrt{\frac{T(\mathrm{E} 2)}{T(\mathrm{M} 1)}}= \pm \frac{\sqrt{3}}{10} \frac{\omega}{C} \sqrt{\frac{B\left(\mathrm{E} 2 \mid I \rightarrow I^{\prime}\right)}{B\left(\mathrm{M} 1 \mid I \rightarrow I^{\prime}\right)}}
$$


where the \pm sign has to be chosen depending on the relative sign of the reduced matrix elements. The electric quadrupole and magnetic dipole transition probabilities $T(\mathrm{E} 2)$ and $T(\mathrm{M} 1)$ are given by

$$
\begin{aligned}
& T\left(\mathrm{E} 2 \mid I \rightarrow I^{\prime}\right)=\frac{4 \pi}{75} \frac{1}{\hbar}\left(\frac{\omega}{C}\right)^{5} B\left(\mathrm{E} 2 \mid I \rightarrow I^{\prime}\right), \\
& T\left(\mathrm{M} 1 \mid I \rightarrow I^{\prime}\right)=\frac{16 \pi}{9} \frac{1}{\hbar}\left(\frac{\omega}{C}\right)^{3} B\left(\mathrm{M} 1 \mid I \rightarrow I^{\prime}\right) .
\end{aligned}
$$

The reduced nuclear transition probability $B(\mathrm{E} 2)$ for the excitation process may be computed from the partial reduced transition probability $\in B(\mathrm{E} 2)$ and the assumed conversion coefficients,

$$
B(\mathrm{E} 2)=\frac{2 I_{\text {down }}+1}{2 I_{\text {up }}+1} R \in B(\mathrm{E} 2)
$$

where $B$ (E2) is in units of $e^{2} \times 10^{-48} \mathrm{~cm}^{4} . R$ is the partial radiation factor and is given by:

$$
R_{j}=\sum_{i} f_{i}\left(1+\alpha_{i}\right) / f_{j} .
$$

Thus, if the detected radiation is a $\gamma$-ray $\left(\gamma_{j}\right)$ the decay fraction is given by

$$
\in\left(\gamma_{j}\right)=f_{j} / \sum_{i}\left(1+\alpha_{i}\right) f_{i},
$$

where $f_{i}$ are the relative intensities of the various gamma transitions by which the excited state may decay, and $\alpha_{i}$ are the corresponding total conversion coefficients.

The reduced transition probability $B(\mathrm{E} 2)$ can be calculated from the transition probability $T(\mathrm{E} 2)$ as

$$
B(\mathrm{E} 2)=\left(81.4 / E_{\gamma}{ }^{5}\right) T_{\gamma}(\mathrm{E} 2),
$$

where $E_{\gamma}$ is in $\mathrm{keV}$ and $B(\mathrm{E} 2)$ in $e^{2} \times 10^{-48} \mathrm{~cm}^{4}$ units, and

$$
T_{\gamma}(\mathrm{E} 2)=\left[T_{1 / 2} \cdot 1.44\left(1+1 / \delta^{2}\right) \cdot R\right]^{-1},
$$

where $E_{\gamma}$ is in $\mathrm{keV}$ and $B(\mathrm{E} 2)$ in $e^{2} \times 10^{-48} \mathrm{~cm}^{4}$ units, and

\section{Results and Discussions}

The knowledge of the level scheme of a nucleus furnishes an important clue to its structure. By level scheme we mean not only the energies of the levels but also the character, that is to say the spin and parity of each level ${ }^{9}$ and the transition probabilities $^{7}$ between any two nuclear levels. During the last few years a great amount of work has been done in this field at our laboratory on even-even Osmium isotopes $7,8,9$. The ground state of an even-even nucleus is $0+$ without any known exception, the first excited state is $2+$ with very few exceptions. The energy El of the first excited state of the Osmium nuclei (76 protons) however, straddles the "energy gap" for the $88 \rightarrow 90$ neutron transition ${ }^{17}$. As to the level scheme of ${ }^{186} \mathrm{Os},{ }^{188} \mathrm{Os},{ }^{190} \mathrm{Os}$ and ${ }^{192} \mathrm{Os}$, interesting work had been done by several groups ${ }^{9,17}$, and the decay schemes are well established. The studies yielded a second $2+$ state at an energy 2.4 times that of the first state in ${ }^{192} \mathrm{Os}$, while the second $2+$ states in the isotopes ${ }^{186} \mathrm{Os}$ and ${ }^{188} \mathrm{Os}$ lie high above the $4+$ states, see Figure 1. There is rather good reason to believe that in ${ }^{190} \mathrm{Os}$ the $2+$ state lies just a little above the $4+$ state.

In order to see the microscopic behaviour of the low-lying collective states, we investigated the E0 and Ml transition $\left(2^{\prime}+\rightarrow 2+\right)$, the penetration effects in Ml transition and the E2/Ml mixing ratios in even-even Osmium nuclei.

The abrupt change of $\delta$ with neutron number, Figure 2 indicates the existance of penetration effects. It is noted from Fig. 2 that the structure for the magnetic dipole transition is obviously quantitatively related ${ }^{17}$ to the structure of the $g_{R}$ factors especially in the $\mathrm{Os}$ region. It should be noted however, that the experimental errors of the $g_{R}$ factors scatter the related predictions for $(\delta / \mathrm{E})^{2}$ appreciably.

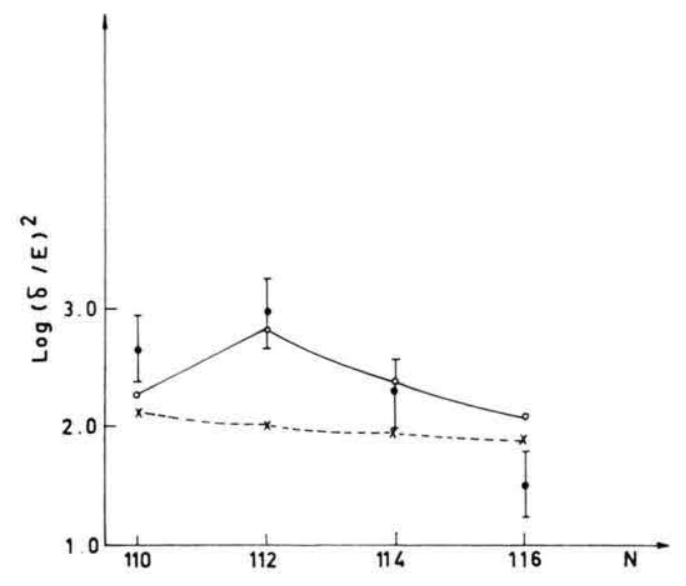

Fig. 2. The values for $\log (\delta / E)^{2}$ for Osmium nuclei. The dotted curve gives theoretical predictions calculated by Greiner ${ }^{17}$ while the full line gives theoretical values for $\log (\delta / E)^{2}$ using the experimental $g_{\mathrm{R}}$ factors.

The results for the adopted values of $B(\mathrm{E} 2$, $0 \rightarrow 2)$ and $B\left(\mathrm{E} 2,2^{\prime} \rightarrow 2\right)$ are compared with the calculations of Kumar and Baranger ${ }^{10}$, Table 1. The 
Table 1. Summary of results for second and higher $2^{\prime}+$ states in ${ }^{186-192} \mathrm{Os}$.

\begin{tabular}{|c|c|c|c|c|c|c|c|c|c|c|c|}
\hline \multirow{2}{*}{ Nucleus } & \multirow{2}{*}{$\begin{array}{l}E \\
\text { Level } \\
(\mathrm{keV})\end{array}$} & \multicolumn{2}{|c|}{$\begin{array}{l}B\left(\mathrm{E} 2,2^{\prime} \rightarrow 2\right) \\
10^{-51} \mathrm{e}^{2} \mathrm{~cm}^{4}\end{array}$} & \multicolumn{2}{|c|}{$\begin{array}{l}B\left(\mathrm{Ml}, 2^{\prime} \rightarrow 2\right) \\
10^{-6}(\mathrm{eh} / 2 \mathrm{Mc})^{2}\end{array}$} & \multicolumn{2}{|c|}{$\begin{array}{l}B\left(\mathrm{E} 2,2^{\prime} \rightarrow\right) 0 / \\
B\left(\mathrm{E} 2,2^{\prime} \rightarrow 2\right)\end{array}$} & \multirow[t]{2}{*}{$\begin{array}{l}T\left(2^{\prime} \rightarrow 2\right) / \\
T\left(2^{\prime} \rightarrow 0\right)\end{array}$} & \multicolumn{2}{|c|}{$\delta$} & \multirow[t]{2}{*}{$a_{\text {tot }}$} \\
\hline & & $\exp$ & KB & $\exp$ & KB & $\exp$ & KB & & $\exp$ & KB & \\
\hline${ }^{186} \mathrm{Os}$ & 768 & $85 \pm 14$ & 256 & $9 \pm 2$ & 328 & $0.450 \pm 0.02$ & 0.148 & $0.85 \pm 0.05$ & \pm 2 & -14.7 & $0.011 \pm 0.001$ \\
\hline${ }^{188} \mathrm{Os}$ & 633 & $161 \pm 14$ & 403 & $30 \pm 7$ & 705 & $0.342 \pm 0.018$ & 0.091 & $0.80 \pm 0.03$ & $15 \pm 0.9$ & -9.5 & $0.025 \pm 0.003$ \\
\hline${ }^{190} \mathrm{Os} \S \S$ & 557 & $270 \pm 20$ & 539 & $362 \pm 251$ & 899 & $0.173 \pm 0.007$ & 0.053 & $0.792 \pm 0.030$ & $8.5 \pm 2$ & -7.6 & 0.0504 \\
\hline${ }^{192} \mathrm{Os}$ & 489 & $338 \pm 41$ & 743 & $1920 \pm 450$ & 1545 & $0.11 \pm 0.006$ & 0.009 & $0.661 \pm 0.05$ & $4.1 \pm 1.0$ & -5.2 & $0.109 \pm 0.009$ \\
\hline
\end{tabular}

$\$$ The results for ${ }^{190} \mathrm{Os}$ have been taken from Reference 16.

results for ${ }^{190} \mathrm{Os}$ have been taken from Coulomb excitation measurements ${ }^{16}$. The magnitudes of the calculated values of $B(\mathrm{E} 2,0 \rightarrow 2)$ agree quite well with the experimental results for all nuclei with a

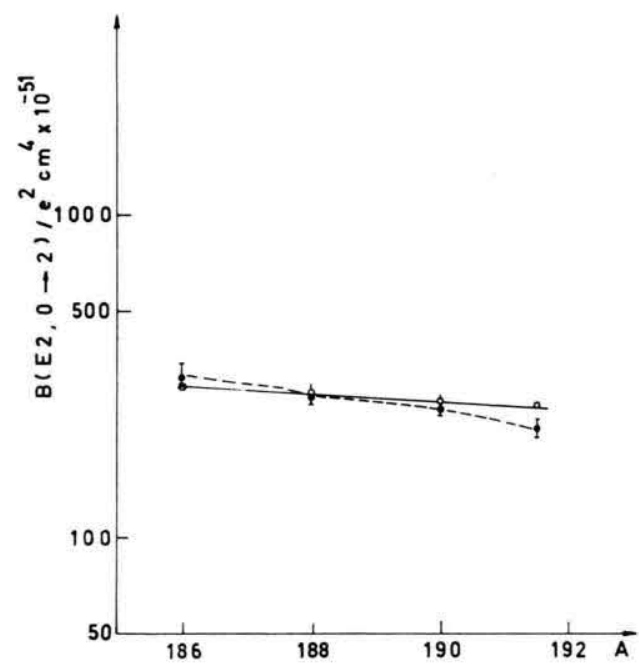

Fig. 3. The experimental reduced transition probabilities $B(\mathrm{E} 2,0 \rightarrow 2)$ in Osmium nuclei. The solid curve is due to Kumar values.

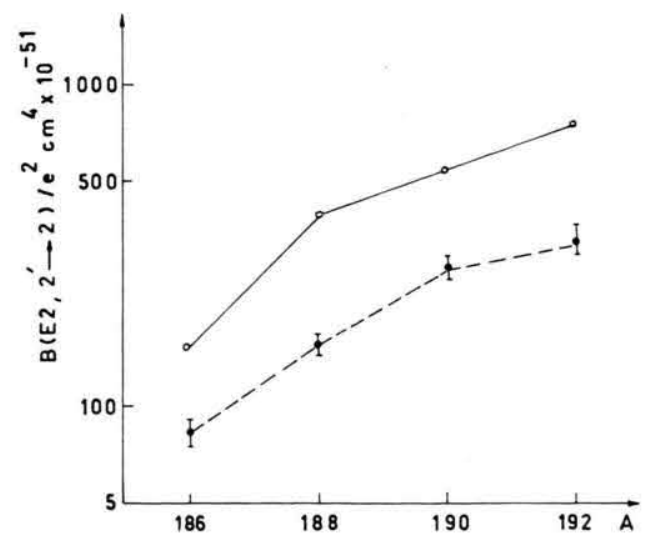

Fig. 4. The experimental reduced transition probabilities $B\left(\mathrm{E} 2,2^{\prime} \rightarrow 2\right)$ in Osmium nuclei. The solid curve is due to Kumar values. possible exception in the case of ${ }^{192} \mathrm{Os}$ where Kumar's value is $30 \%$ larger than the experimental value, see Figure 3.

A summary of the properties of second and higher $2^{\prime}+$ states are given in Table 1 , along with the calculated results of Kumar and Baranger ${ }^{10}$. The large disagreements in the values of $B\left(\mathrm{E} 2,2^{\prime} \rightarrow 2\right)$ are observed in Fig. 4, where the calculated values are larger than the experimental ones. The values of the ratios $B\left(\mathrm{E} 2,2^{\prime} \rightarrow 0\right) / B\left(\mathrm{E} 2,2^{\prime} \rightarrow 2\right)$ shown in Fig. 5 decrease sharply from 0.46 to 0.12 over the region ${ }^{186-192} \mathrm{Os}$. Also, the large deviations found in the reduced magnetic dipole transition probabilities $B\left(\mathrm{M} 1,2^{\prime} \rightarrow 2\right)$, see Table 1 , indicate an existance of some nuclear penetration effects in the Ml transitions.

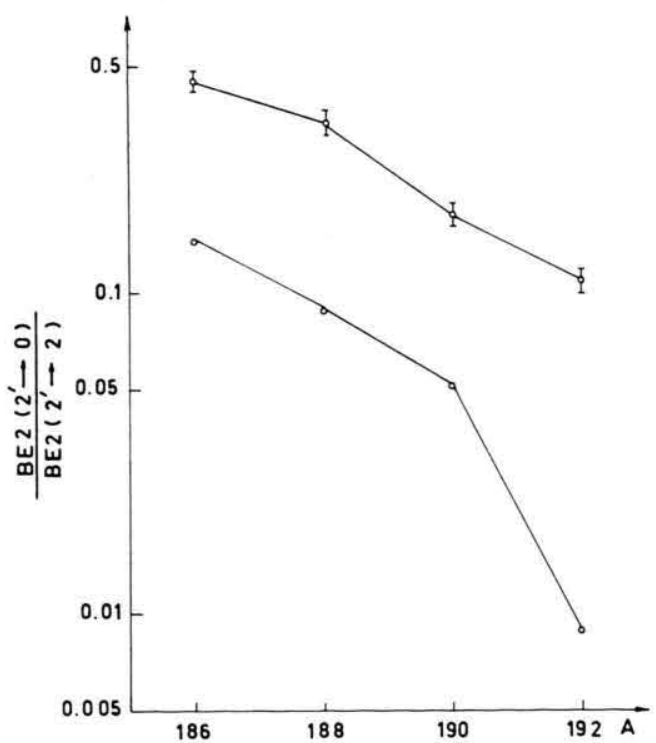

Fig. 5. The experimental reduced transition probabilities ratios $B\left(\mathrm{E} 2,2^{\prime} \rightarrow 0\right) / B\left(\mathrm{E} 2,2^{\prime} \rightarrow 2\right)$ in Osmium nuclei. The lower curve is due to Kumar's calculations. 
The model calculations of Kumar and Baranger ${ }^{10}$ have predicted a prolate shape for ${ }^{186-188} \mathrm{Os}$ and a symmetric shape for ${ }^{190-192} \mathrm{Os}$. The poor agreement between the $\delta$-values for these nuclei may be due to

1 H. Ikegami, K. Sugiyama, T. Yamazaki, and M. Sakai, Nucl. Phys. 41, 30 [1963].

2 M. Sakai, M. Nozawa, H. Ikegami, and T. Yamazaki, Nucl. Phys. 53, 529 [1964].

3 T. Hirose, S. Morinobu, and H. Ikegami, Nucl. Phys. A 146, 220 [1970]

4 T. R. Gerholm, M. S. El-Nesr, E. Bashandy, and B.-G. Pettersson, Ark. Fysik 21, 241 [1962].

5 E. Bashandy, Z. Naturforsch. 26 a, 1109 [1971].

6 E. Bashandy and M. S. El-Nesr, Nucl. Phys. 31, 177 [1962].

7 E. Bashandy and M. S. El-Nesr, Nucl. Phys. 34, 483 [1962].

8 M. S. El-Nesr and E. Bashandy, Physica 28, 1335 [1962]. penetration effects in the calculated Ml matrix elements and the predictions of Kumar and Baranger for this group of nuclei may well be fulfilled.

${ }^{9}$ E. Bashandy and S. G. Hanna, Nucl. Phys. 84, 577 [1966].

$10 \mathrm{~K}$. Kumar and M. Baranger, Nucl. Phys. A 122, 273 [1968].

11 K. Kumar, Phys. Lett. 28 B, 25 [1969].

12 E. L. Church and J. Weneser, Phys. Rev. 103, 1035 [1956].

13 G. Scharff-Goldhaber and J. Weneser, Phys. Rev. 98, 212 [1955].

14 L. Wilets and M. Jean, Phys. Rev. 102, 788 [1956].

15 S. Hultberg, B. Nagel, and P. Olsson, Ark. Fysik 20, 555 [1961].

16 W. T. Milner, F. K. McGowan, R. L. Robinson, P. H. Stelson, and R. O. Sayer, Nucl. Phys. A 177, 1 [1971].

17 W. Greiner, Nucl. Phys. 80, 417 [1966]. 